\title{
Comparative metabolomics analysis reveals the variations of eating quality among three high-quality rice cultivars
}

\author{
Rong Gong • Daoqiang Huang • Yibo Chen • Hong Li • Zhidong Wang • Degui Zhou • \\ Lei Zhao • Yangyang Pan • Yuxiao Chang • Yong Xiang • Chongrong Wang • \\ Shaochuan Zhou
}

Received: 12 July 2020 / Accepted: 26 November 2020 /Published online: 10 December 2020

(C) The Author(s) 2020

\begin{abstract}
Good eating quality is a highly desirable trait of rice which determines its commercial value and market share. However, the molecular basis of this trait remains largely unknown. Here, three high-quality conventional rice cultivars, including two superior eating quality cultivars Meixiangzhan-2 (MXZ) and Xiangyaxiangzhan (XYXZ), and one ordinary eating quality cultivar Huanghuazhan (HHZ), were analyzed by comparative metabolomics to identify the inherent mechanism for the formation of superior eating quality. The results showed $58.8 \%$ of common differential substances between MXZ vs HHZ and XYXZ vs HHZ were enriched in MXZ and XYXZ, whereas $39.2 \%$ of them were prominently decreased compared with HHZ, mainly including amino acids, carbohydrates, lipids, phenolamides, and flavonoids, which may be the primary factors leading to the differences of taste and flavor among these three cultivars. We also found that lysine derivatives and fatty acids may have a close relationship with taste. These results above provide important insights into the taste-forming mechanism of rice and will be beneficial for superior eating quality rice breeding.
\end{abstract}

R. Gong $\cdot$ D. Huang $\cdot$ Y. Chen $\cdot$ H. Li $\cdot$ Z. Wang •

D. Zhou $\cdot$ L. Zhao - Y. Pan $\cdot$ C. Wang $(\square) \cdot$ S. Zhou $(\bowtie)$

Guangdong Key Laboratory of New Technology in Rice

Breeding, The Rice Research Institute of Guangdong Academy of Agricultural Sciences, Guangzhou 510640, China

e-mail: wangcr1980@163.com

e-mail: xxs123@163.com

Y. Chang $\cdot$ Y. Xiang

Agricultural Genomics Institute at Shenzhen, Chinese Academy of Agricultural Sciences, Shenzhen 518120, China
Keywords High-quality rice $\cdot$ Metabolomics . Differentially accumulated metabolites (DAMs) . Superior eating quality $\cdot$ Nutrient quality

\section{Introduction}

Rice is the principal food for over half of the population of the world. The rice quality directly affects its commercial value and market position (Fitzgerald et al. 2009). Therefore, on the basis of high and stable yield, improving rice quality has become a new goal of rice breeding in China. With the continuous development of marker-assisted selection (MAS) breeding technology, more and more high-quality rice cultivars enter the market. Due to the particularity of cultivars, superior eating quality rice are widely welcomed by consumers because of its unique taste and flavor, and their prices are $25 \%$ or even several times higher than that of general eating quality cultivars. However, the existing highquality cultivars are unable to meet the growing demand of rice industry on account of the low breeding efficiency. Hence, it is an urgent need to illuminate the formation mechanism of eating quality and explore the feature compounds of taste and flavor for new superior eating quality cultivar breeding.

The evaluation modes of rice eating quality are diversified, mainly by the sensory perception (Takeuchi et al. 2008), whereas the mechanism of it is very complex, which could be affected by the processing, cooking, nutritional and appearance quality at same time (Chen et al. 2005; Baxter et al. 2010; Kang et al. 2011), leading to slow 
progress in the study of its molecular mechanism. Currently, the research of rice eating quality are mainly focused on the effect of starch content (Li et al. 2016), protein content (Wakamatus et al. 2008; Champagen et al. 2009), fat content (Gu et al. 2011), cultivation mode and environment interaction (Zhang et al. 2009; Zhang et al. 2012), and a lot of functional genes relevant to taste have been found. For example, $W x$ controls amylose content and gel consistency and has many alleles in different cultivars (Tian et al. 2009). Recently, the domestication relationship of them has been clarified with the discovery of their ancestral allele $W x^{l v}$ (Zhang et al. 2019); glutelin type-A2 precursor $(O s G l u A 2)$ regulates protein content (Yang et al. 2019); lipid transport protein 36 (OsLTP36) involves in lipid transport (Wang et al. 2015); and lipidoxidase ( $L O X)$ mediates the content of unsaturated fatty acid (Gayen et al. 2015) and so on. Nevertheless, there are few studies on the role of metabolites in the formation of eating quality. Metabolites are the end products of cell regulatory process, of which the category and quantity changes are regarded as the final response of biological system to genes or environment ( $\mathrm{Lu}$ et al. 2013). The study of plant metabolome plays important roles in understanding plant metabolic pathway to enhance plant adaptive ability to environmental stress (Isshiki et al. 2014; Nakabayashi and Saito 2015), improving agronomic characters, and thus increasing crop yield (Gong et al. 2013). By combining with other omics technology, metabolomics also could exploit the characteristic compounds in the process of plant development and illuminate genetic mechanism. For example, the hereditary basis of tomato flavor was first demonstrated by metabolomics and genomics association analysis and 50 gene loci for flavor substances were obtained (Tieman et al. 2017); combined with proteomics, researchers revealed that the main reason for the decrease of alfalfa nutritional quality in the flowering stage was the content change of L-glutamic acid, L-phenylalanine, and carbohydrate metabolism-related substances in the leaves (Fan et al. 2018); through the metabolomics and transcriptomics, more than 16 kinds of anthocyanin and related genes were identified from asparagus (Dong et al. 2019). In recent years, metabolomics has been also reported to analyze different rice characters. By using the genome-wide association analysis of the metabolomics and phenotypes from 502 rice cultivars, researchers revealed the natural variation and genetic control of various metabolites in rice grain and explored a few metabolites related to grain shape, grain color, and rice nutritional quality (Chen et al. 2016). In addition, the yellow mechanism of milled rice and the contribution factors of aroma were also discussed by metabolomics (Concepcion et al. 2018; Liu et al. 2019), but little research about rice taste and flavor was reported.

Huanghuazhan (HHZ), Meixiangzhan-2 (MXZ), and Xiangyaxiangzhan (XYXZ) are all high-quality conventional rice cultivars with widely cultivated area in South China. In them, only MXZ and XYXZ have fragrant and are awarded the golden medal in the eating quality evaluation contest of national high-quality rice in China. MXZ and XYXZ are also widely accepted by farmers and rice industry as superior eating quality cultivars, while HHZ was only recognized as a high-yield cultivar with ordinary eating quality. In order to clarify the genetic mechanism of eating quality in these three cultivars, the physical and chemical indices of rice quality were tested and little difference among them was found. Comparative metabolomics analysis demonstrated that there are significant differences of amino acids, phenolamides, flavonoids, lipids, and carbohydrates in MXZ and XYXZ compared with HHZ. We speculated these compounds were closely related to the formation of the rice taste and flavor. These results would provide an important theoretical base for exploiting feature compound markers of taste and benefit to superior highquality rice breeding development.

\section{Materials and methods}

\section{Plant materials}

Huanghuazhan (HHZ), Meixiangzhan-2 (MXZ), and Xiangyaxiangzhan (XYXZ) were grown under natural field conditions at the experimental station of Guangdong Academy of Agricultural Sciences $\left(113.30^{\circ} \mathrm{E}\right.$, $23.20^{\circ} \mathrm{N}$ ), Guangzhou, China. The mature seeds of these three cultivars were harvested at the 30 days after flowering and placed in liquid $\mathrm{N}_{2}$ immediately, then stored at $-80{ }^{\circ} \mathrm{C}$ until further analysis. The browned rice samples were sent to Wuhan Metware Biotechnology Co., Ltd. (Wuhan, China) for metabolite profiling analysis with a widely targeted metabolomics method (Chen et al. 2013).

The physical and chemical quality determination of rice

The harvested rice grains were dried and sent to Rice Product Quality Inspection \& Supervision Testing Center of MOA for physical and chemical quality 
determination with the evaluation standard for rice quality by Ministry of Agriculture, China. The contents of determination include grain length, grain width, lengthwidth ratio, chalkiness degree, chalky rice rate, clarity, alkali value, gel consistency, amylose content, protein content, taste value, and aroma. For alkali value detection, six intact milled rice were added with $10 \mathrm{~mL}$ $0.304 \mathrm{~mol} / \mathrm{L} \mathrm{KOH}$ and cultured in $30{ }^{\circ} \mathrm{C}$ incubator for $23 \mathrm{~h}$. Then, the digestion of endosperm was observed and scored according to NY/T 83-2017; for gel consistency measuring, $88 \mathrm{mg}$ flour was taken from $10 \mathrm{~g}$ crushed milled rice (sieved by $0.15 \mathrm{~mm}$ griddle) and added $0.2 \mathrm{~mL}$ thymol blue and $2.0 \mathrm{~mL} 0.2 \mathrm{~mol} / \mathrm{L} \mathrm{KOH}$ successively for mixing, then boiled in water for $8 \mathrm{~min}$, cooled at room temperature for $5 \mathrm{~min}$, ice water bath for $20 \mathrm{~min}$, and then the rice glue length was determined after horizontal standing in $25^{\circ} \mathrm{C}$ incubator for $1 \mathrm{~h}(\mathrm{~GB} /$ $\mathrm{T}$ 22294-2008). The amylose and protein contents were detected as methods GB/T 15683-2008 and GB 5009.52016, respectively. The taste value was measured by rice taste meter SATAKE/STA1B after cooking (with 1:1.2 rice/water), and aroma was evaluated by sensory perception. The detected methods for other test indexes were referred to NY/T 83-2017.

Sample preparation and extraction for metabolomics analysis

The freeze-dried seeds were crushed using a mixer mill (MM 400, Retsch) with a zirconia bead for $1.5 \mathrm{~min}$ at $30 \mathrm{~Hz}$. One-hundred-milligram powder was weighted and extracted overnight at $4{ }^{\circ} \mathrm{C}$ with $1.0 \mathrm{~mL} 70 \%$ aqueous methanol. Following centrifugation at $10,000 \mathrm{~g}$ for $10 \mathrm{~min}$, the extracts were absorbed (CNWBOND Carbon-GCB SPE Cartridge, $250 \mathrm{mg}$, $3 \mathrm{~mL}$; ANPEL, Shanghai, China, www.anpel.com. $\mathrm{cn} / \mathrm{cnw}$ ) and filtrated (SCAA-104, $0.22 \mu \mathrm{m}$ pore size; ANPEL, Shanghai, China, http://www.anpel.com.cn/) before LC-MS analysis.

High-performance liquid chromatography conditions

For each cultivar, three biological replicates were independently analyzed. The sample extracts were analyzed using an LC-ESI-MS/MS system (HPLC, Shim-pack UFLC SHIMADZU CBM30A system, www. shimadzu.com.cn/; MS, Applied Biosystems 6500 Q TRAP, www.appliedbiosystems.com.cn/). The analytical conditions were as follows: HPLC: column,
Waters ACQUITY UPLC HSS T3 C18 $(1.8 \mu \mathrm{m}, 2.1$ $\mathrm{mm} * 100 \mathrm{~mm})$; solvent system, water $(0.04 \%$ acetic acid): acetonitrile ( $0.04 \%$ acetic acid); gradient program, 95:5 V/V at $0 \mathrm{~min}, 5: 95 \mathrm{~V} / \mathrm{V}$ at $11.0 \mathrm{~min}, 5: 95 \mathrm{~V} / \mathrm{V}$ at $12.0 \mathrm{~min}, 95: 5 \mathrm{~V} / \mathrm{V}$ at $12.1 \mathrm{~min}, 95: 5 \mathrm{~V} / \mathrm{V}$ at $15.0 \mathrm{~min}$; flow rate, $0.40 \mathrm{~mL} / \mathrm{min}$; temperature, $40{ }^{\circ} \mathrm{C}$; injection volume: $2 \mu \mathrm{L}$. The effluent was alternatively connected to an ESI-triple quadrupole-linear ion trap (Q TRAP)MS.

Quality control (QC) samples were prepared from all the sample extracts to become a combined sample, then divided into three QC samples and analyzed using the same method as for the experimental samples. The QC samples were injected every three experimental samples throughout the analytical run to provide a set of data from which repeatability could be assessed.

\section{ESI-Q TRAP-MS/MS}

LIT and triple quadrupole (QQQ) scans were acquired on a triple quadrupole-linear ion trap mass spectrometer (Q TRAP), API 6500 Q TRAP LC/MS/MS System, equipped with an ESI Turbo Ion-Spray interface, operating in a positive ion mode and controlled by Analyst 1.6 software (AB Sciex). The ESI source operation parameters were as follows: ion source, turbo spray; source temperature $500{ }^{\circ} \mathrm{C}$; ion spray voltage (IS) $5500 \mathrm{~V}$; ion source gas I (GSI), gas II (GSII), curtain gas (CUR) were set at 55, 60, and 25.0 psi, respectively; the collision gas (CAD) was high. Instrument tuning and mass calibration were performed with 10 and $100 \mu \mathrm{mol} / \mathrm{L}$ polypropylene glycol solutions in QQQ and LIT modes, respectively. QQQ scans were acquired as MRM experiments with collision gas (nitrogen) set to 5 psi. DP and CE for individual MRM transitions was done with further DP and CE optimization. A specific set of MRM transitions were monitored for each period according to the metabolites eluted within this period.

Qualitative and quantitative analysis of metabolites

Qualitative analysis of primary and secondary MS data was carried out by comparison of the accurate precursor ions (Q1), product ions (Q3) values, the retention time (RT), and the fragmentation patterns with those obtained by injecting standards using the same conditions if the standards were available (Sigma-Aldrich, USA http:/www.sigmaaldrich.com/united-states.html) or conducted using a self-compiled database MWDB 
(MetWare biological science and Technology Co., Ltd. Wuhan, China) and publicly available metabolite databases if the standards were unavailable. Repeated signals of $\mathrm{K}^{+}, \mathrm{Na}^{+}, \mathrm{NH}_{4}{ }^{+}$, and other large molecular weight substances were eliminated during identification. The quantitative analysis of metabolites was based on the MRM mode. The characteristic ions of each metabolite were screened through the QQQ mass spectrometer to obtain the signal strengths. Integration and correction of chromatographic peaks was performed using MultiQuant version 3.0.2 (AB SCIEX, Concord, Ontario, Canada). The corresponding relative metabolite contents were represented as chromatographic peak area integrals.

\section{qRT-PCR analysis}

Total RNA of mature shelled seeds (30 days after heading) was extracted with a Quick RNA Isolation Kit (Cat. \#: 0416-50 GK; Huayueyang Biotech, https://www. huayueyang.com.cn) according to the manufacturer's instructions. A total of $1 \mu \mathrm{g}$ of RNA was used to synthesized cDNA with the TransScript ${ }^{\circledR}$ One-step gDNA removal and cDNA Synthesis Super Mix (TransGen Biotech, https://www.transgen.com.cn). The expression of metabolism-related genes was detected using specific primers listed in Table S6. qRT-PCR was conducted using TransStart Tip Green qPCR SuperMix (TransGen Biotech, https://www.transgen.com.cn). For each sample, qRT-PCR was performed with three technical and three biological replicates. The rice ubiquitin (UBQ) gene was used as the internal control, and expression levels of the examined genes were analyzed via the $2^{-\Delta \Delta \mathrm{CT}}$ method (Livak and Schmittgen 2001).

\section{Statistical analysis}

Metabolite data analysis was conducted with the Analyst 1.6.3 software. The supervised multivariate method, orthogonal partial least squares-discriminant analysis (OPLS-DA), was used to maximize the metabolome differences between the pair of samples. The relative importance of each metabolite to the OPLS-DA model was checked using the parameter called variable importance in projection (VIP). Metabolites with VIP $\geq 1$ and fold change $\geq 2$ or $\leq 0.5$ were considered differential metabolites for group discrimination (Saccenti et al. 2013). Other data were statistically analyzed using the SPSS 20.0 statistical package and presented as the mean \pm standard deviation ( \pm SD). The differences between the groups were estimated using an analysis of variance, and $P \leq 0.05$ was considered to indicate a statistically significant difference between the two groups.

\section{Results}

The physicochemical index analysis of grain quality among three rice cultivars

To elucidate the differences of the high-quality rice cultivars, HHZ, XYXZ, and MXZ, which were the representative indica cultivars with good quality and planted widely in South China, were selected to study. Twelve kinds of mainly physicochemical indices used for grain quality evaluation currently were determined in these three cultivars, including grain length, grain width, length-width ratio, chalkiness degree, chalky grain rate, and clarity as appearance quality, and alkali value, gel consistency, aroma, taste value, amylose, and protein content as internal quality. Our results showed that in terms of appearance quality, XYXZ has the smallest grain width and the largest grain length, resulting in the largest length-width ratio (Fig. 1a, S1a). HHZ possesses the highest chalkiness degree and lowest chalky grain rate, whereas the chalkiness degree $(0.5 \%)$ in MXZ is the lowest, while the chalkiness rate is higher than HHZ (5\%). In terms of internal quality, the highest protein content is in XYXZ, while HHZ has the highest amylose content and gel consistency. MXZ and XYXZ have fragrance; however, HHZ does not. Other indices such as clarity, alkali value, and eating value were almost the same among the three cultivars (Table S1). However, on the overall level, the differences of these feature indices above are not significant to distinguish the true rice quality level, especially in eating quality, between these cultivars. Therefore, more effective methods including metabolomics should be performed to uncover the inherent mechanism for the formation of superior eating quality in MXZ and XYXZ.

Widely targeted metabolite profiling of these three rice cultivars

To explore the eating quality differences of these three cultivars, a widely targeted metabolomics approach (UPLC-MS/MS testing) was used to analyze global 
metabolic variations of each rice sample. A total of 547 metabolites were identified among all samples, including 80 amino acids, 76 organic acids, 70 lipids, 45 nucleotides, 18 vitamins, 16 carbohydrates, 215 secondary metabolites (composing flavonoids, hydroxycinnamoyl derivatives, phenolamides, alcohols, alkaloids, etc.), and 27 other metabolites. Moreover, the amount of flavonoids in secondary metabolites was the highest (about 60\%) (Table S2). Principal component analysis (PCA) was performed on samples, including quality control sample (QC, mix of all samples), to provide an initial understanding of the overall metabolic differences between groups of samples and the degree of variability between samples within the group. The results showed that the explained values of PC1 and PC2 for metabolic variances of all samples were $38.95 \%$ and $22.74 \%$, respectively. According to the PCA score, the metabolites of the three cultivars can be divided into three obviously separated sample groups, which indicated that there were large differences in metabolite composition. In addition, the samples in the group were aggregated together with less variation (Fig. 1b). Then, data was normalized and all samples were analyzed with a clustered heat map. The results indicated that the metabolite abundance of three cultivars revealed remarkable variations consistently with PCA data (Fig. 1c). Although there were some differences within the groups, the correlation analysis showed that the correlation coefficient of samples within the group (close to 1) was much higher than that between groups (Fig. S2).

Analysis of differential metabolites among these three cultivars

To provide a deep overview of the metabolic variations, pairwise comparisons with 547 metabolites were carried out between the three cultivars by applying the supervised OPLS-DA model to identify the significant metabolites that were contributed to eating quality. The differentially accumulated metabolites (DAMs) between pair of samples were determined based on variable importance in projection (VIP) $\geq 1$ and the fold change $\geq 2$ or $\leq 0.5$. For each pairwise comparison, 86 metabolites were important in distinguishing MXZ and HHZ, in which 59 and 27 metabolites were predominantly accumulated in MXZ and HHZ, respectively (Fig. 2a, b); 109 metabolites were important to separate XYXZ from HHZ, in which 43 and 66 metabolites were predominantly increased in XYXZ and HHZ, respectively (Fig. 2c, d); there are 71 differential metabolites between XYXZ and MXZ, and 13 and 58 metabolites were significantly enriched in XYXZ and MXZ, respectively (Fig. 2e, f). All of the DAMs were assigned to various metabolic categories according to Kyoto Encyclopedia of Genes and Genomes (KEGG) annotations, including amino acids, flavanoids, flavone C-glycosides, organic acids, lipids, carbohydrates, phenolamides, nucleotides, and others. In these categories, the number of DAMs had obvious difference between HHZ and MXZ, especially flavone C-glycosides and lipids significantly accumulated in MXZ, while other flavanoids enriched in HHZ (Fig. 2b); compared with HHZ, the number of DAMs was similar in XYXZ except flavanoids and lipids, but we found flavanoid and lipid accumulation patterns are consistent with group MXZ vs HHZ (Fig. 2d). Flavanoids were also the most significant category between MXZ and XYXZ, while surprisingly lipids were indiscriminate between them (Fig. 2e). In addition, we found amino acids were prominently enriched in MXZ compared with XYXZ (Fig. 2e).

Comparative analysis of the three groups of DAMs among the three cultivars resolved to 13 common metabolites (Fig. 3a). Of these, 12 metabolites are flavanoids. Correspondingly, the primary enriched KEGG pathway for the DAMs detected among three groups is also the flavone and flavonol biosynthesis (Fig. 3b-d). Besides, we also found that DAMs focused on lysine biosynthesis, thyrold hormone synthesis, and carbohydrate metabolism pathway in MXZ vs HHZ and XYXZ vs HHZ groups (Fig. 3b, c). The DAMs among the three cultivars are listed in Table S3.

Variation levels of metabolites in the flavone and flavonol metabolism

According to the pathway enrichment analysis, representative metabolites participated in flavone and flavonol metabolism are shown in Fig. 4, and fourteen metabolites were detected by widely targeted metabolomics. We found that apigenin and keampferol had not been detected, which are the initial substrates for flavone and flavonol biosynthesis, but two precursors of them, narigenin chalcone, are predominantly accumulated in HHZ and MXZ, while the narigenin is enriched only in HHZ (Fig. 4). In addition, nine flavone derivatives of apigenin were explored from five different metabolic pathways under various enzymatic catalytic reactions. Of these, the 
a
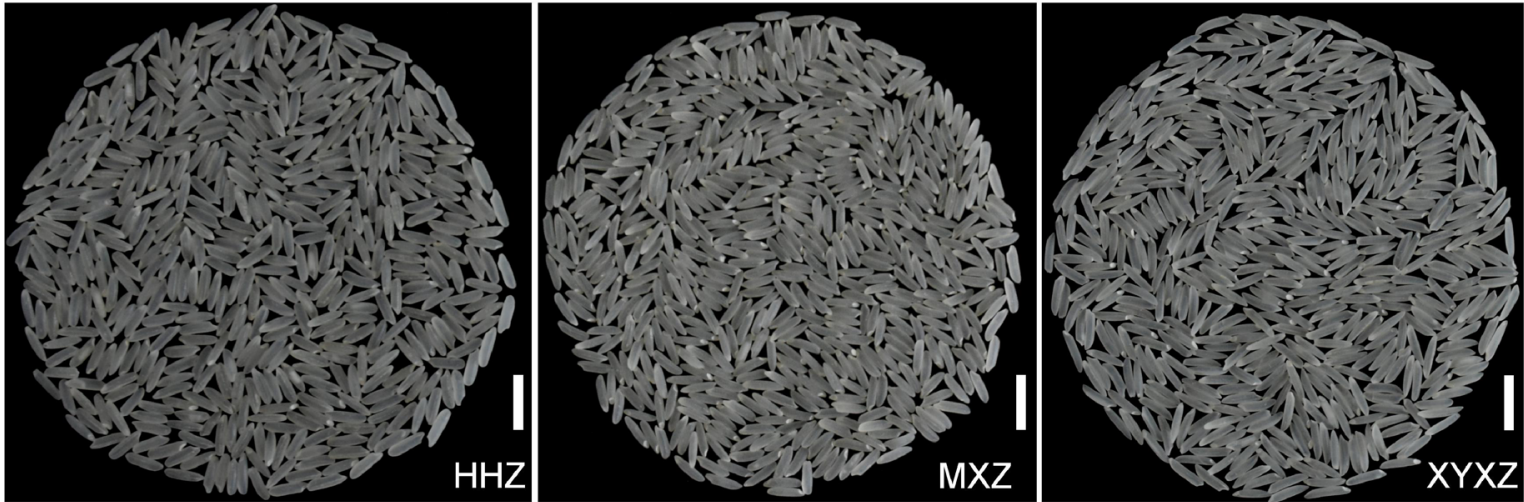

b

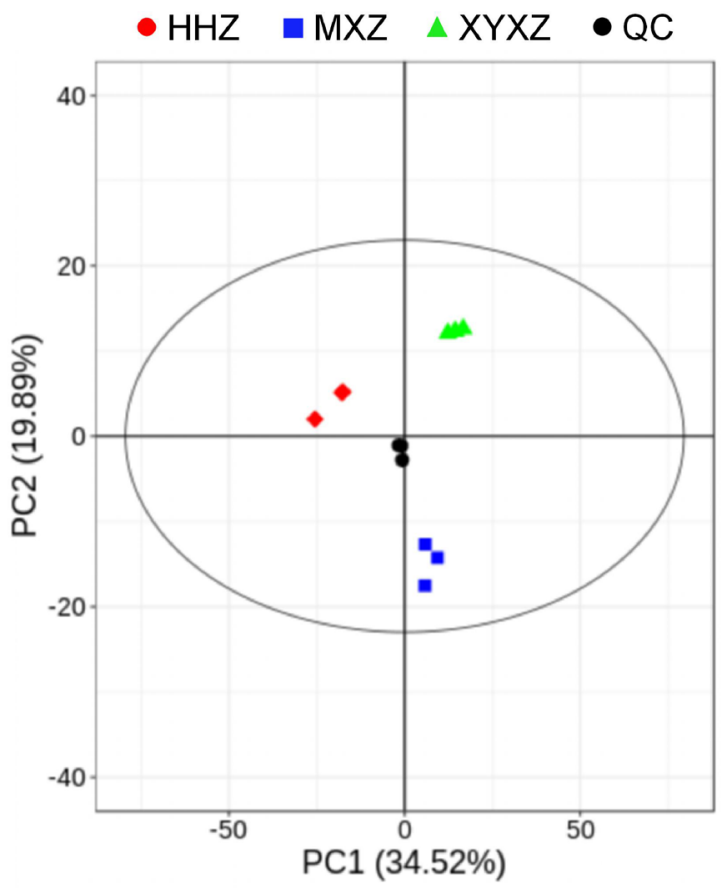

Fig. 1 Widely targeted metabolite profiling identified the metabolites in the test rice. a A polished rice appearance of HHZ, MXZ, and XYXZ. Scale bar: $1 \mathrm{~cm}$. b The principal component analysis

metabolites in three pathways for flavone-C-glycosides biosynthetic were prominently accumulated in MXZ and $\mathrm{XYXZ}$, such as isovitexin, apigenin 6-C-pentoside, apigenin 6-C-hexosyl-8-C-hexosyl-O-hexoside, vitexin 2"-O-beta-L-rhamnoside etc., while the other product contents of flavones, for example, apigenin 7-O-glucoside, apigenin 7-O-neohesperidoside, luteolin 7-O-glucoside, and chrysoeriol, were significantly decreased in
C

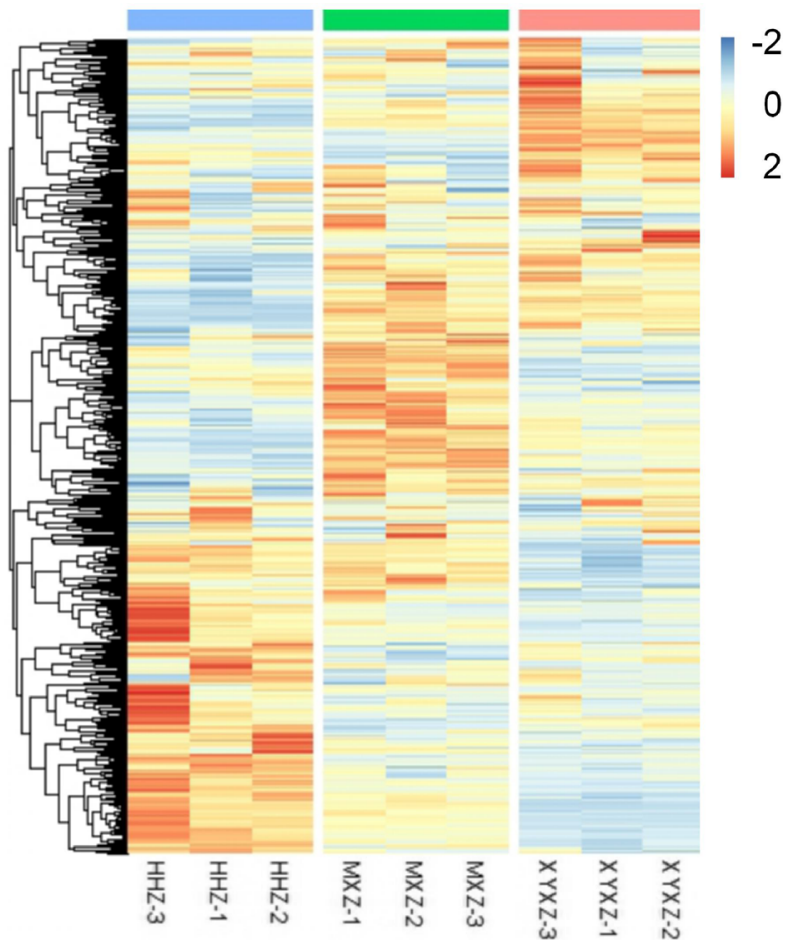

(PCA) of metabolic datasets in three different cultivars. $\mathbf{c}$ A heat map of the relative differences in metabolites among the three rice cultivars $(n=3)$

them, especially in XYXZ. Moreover, we also found that the flavonol derivatives of keampferol are also with lower content in MXZ and XYXZ, like astragalin and trifolin (Fig. 4). These observations above indicated that the metabolites of flavone C-glycosides were accumulated in MXZ and XYXZ in flavone and flavonol metabolism, while other flavone or flavonol compounds decreased significantly. 


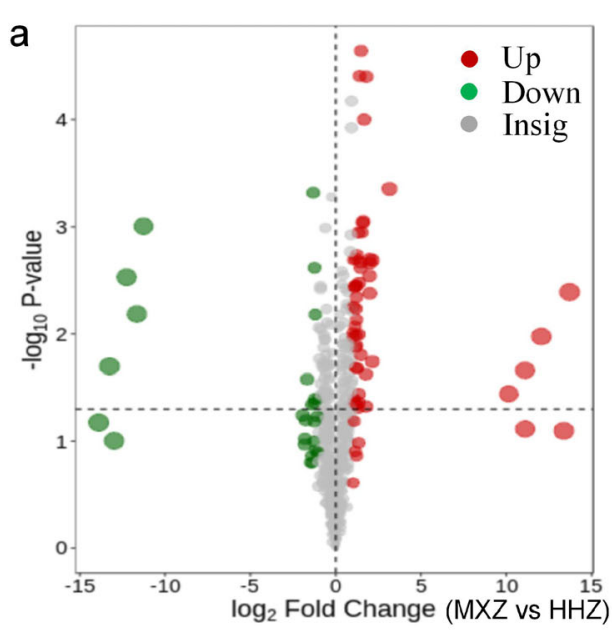

C
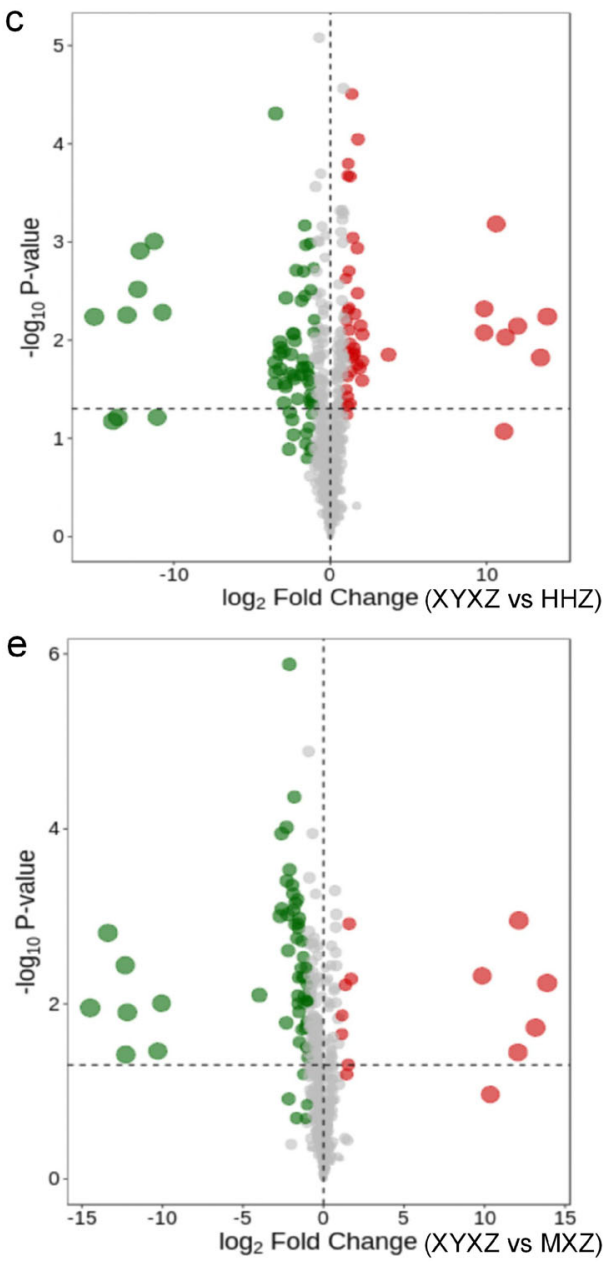

Fig. 2 Identification of differential accumulated metabolite (DAM) analysis among HHZ, MXZ, and XYXZ. a, c, e The volcano plot shows the differential metabolite expression levels between HHZ and MXZ (a), HHZ and XYXZ (c), MXZ and XYXZ (e). Green and red dots represent metabolites with fold

f
Metabolite predominantly accumulated in $\mathrm{MXZ}$

Metabolite predominantly accumulated in $\mathrm{HHZ}$

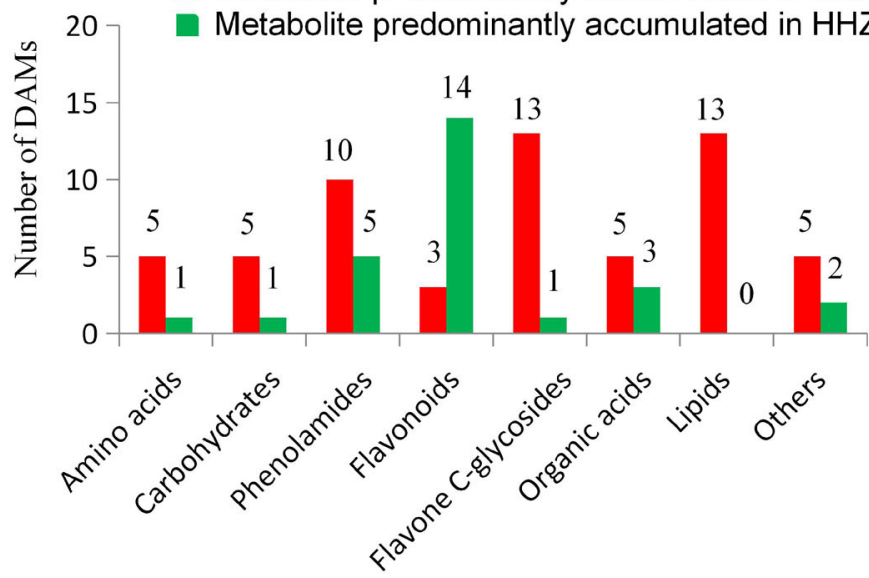

d Metabolite predominantly accumulated in XYXZ

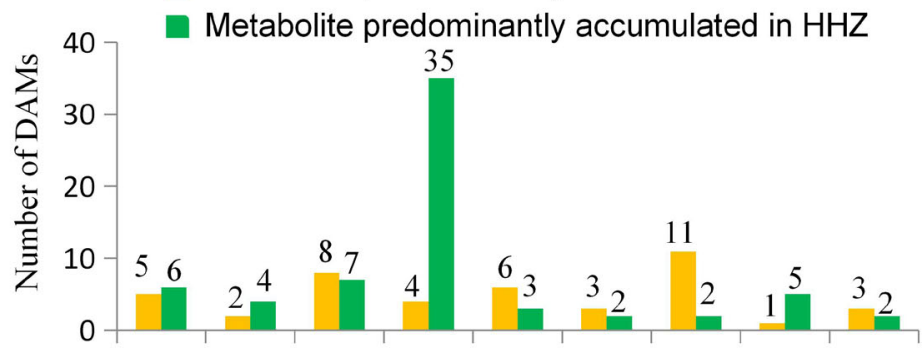

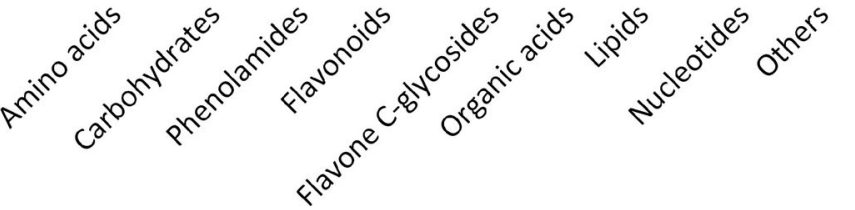
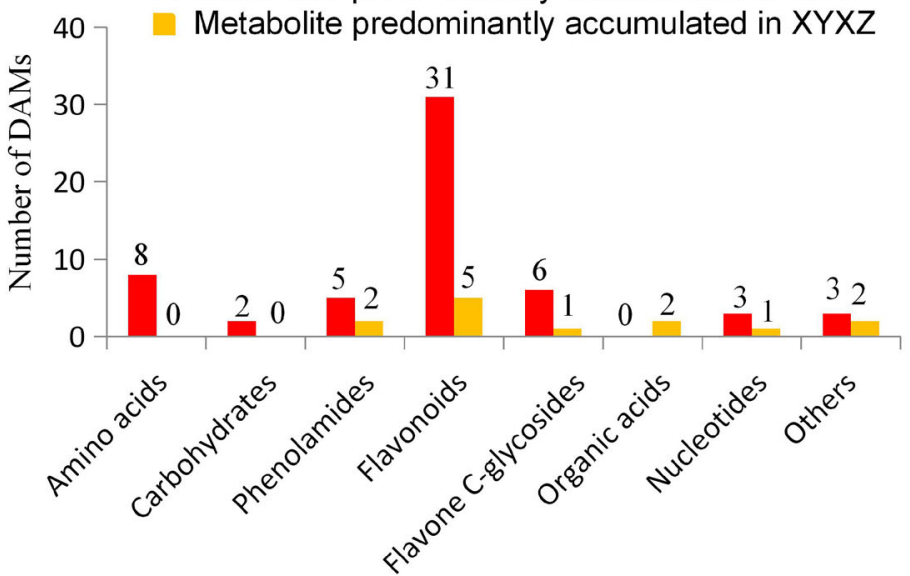

change $\geq 2$ or $\leq 0.5$. The size of dot represents the value of VIP and VIP $\geq 1$. b, d, $\mathbf{f}$ The numbers of predominantly accumulated metabolites in each metabolic category as MXZ compared with HHZ (b), XYXZ compared with HHZ (d), and XYXZ compared with MXZ (f) 
a

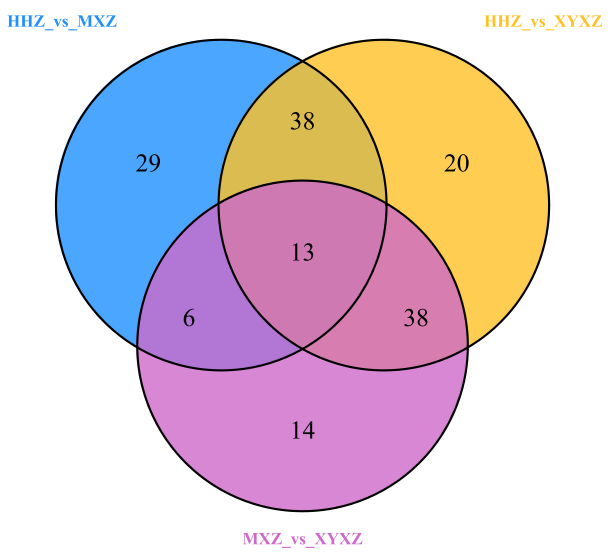

C

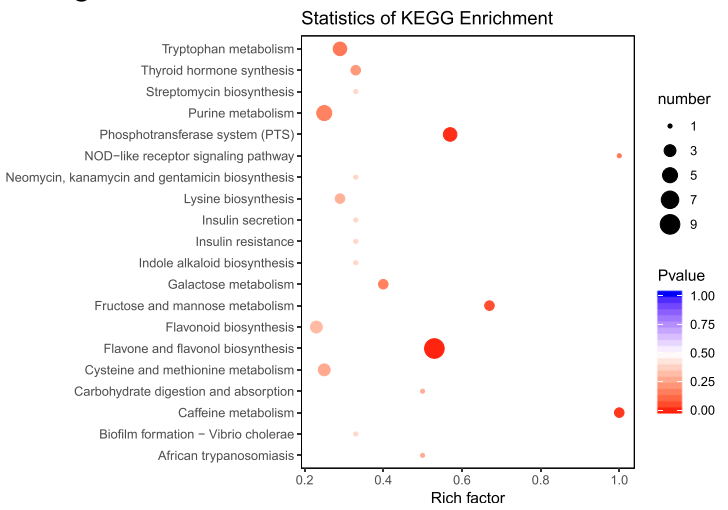

Fig. 3 KEGG pathways enriching analysis of DAMs in three different cultivars. a The Venn diagram shows the overlapping and cultivar-specific DAMs in HHZ, MXZ, and XYXZ. b-d

Variation levels of metabolites in lysine metabolism

As an essential amino acid, lysine is beneficial to human health. To explore the variation of lysine in three cultivars, the lysine metabolism process was discussed in our study (Fig. 5). The results showed that L-aspartic acid was predominantly accumulated both in MXZ and XYXZ, while its downstream metabolites 2, 6diaminoheptanedioate and L-lysine, were enriched in HHZ. As the derivatives of L-lysine, N6-Acetyl-L-lysine, L-pipecolate, and L-saccharopine were more abundant in MXZ compared with others, while allysine, 2aminoadipic acid, and oxoadipic acid were prominently increased both in MXZ and XYXZ (Fig. 5). Moreover, in another metabolic pathway, 5-aminovaleric acid and glutaric acid were enriched only in XYXZ (Fig. 5). Overall, these results suggest that lysine was with the highest level of content in HHZ, but its downstream b

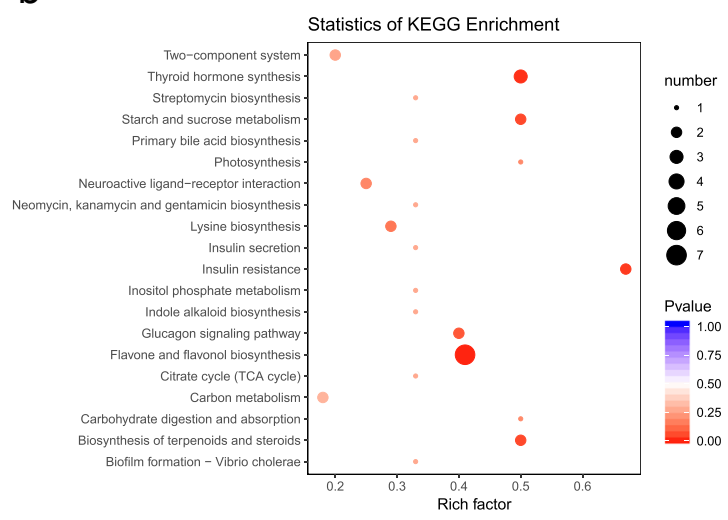

d

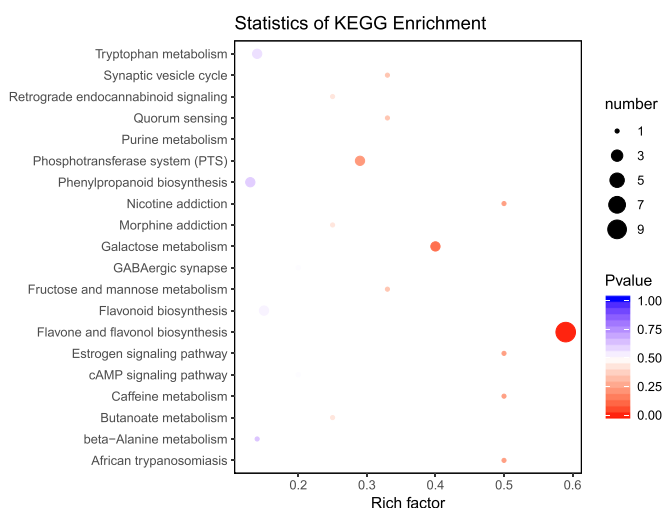

Enrichment of the DAMs between MXZ and HHZ (b), XYXZ and HHZ (c), MXZ and XYXZ (d) in distinct KEGG pathway

metabolites were mainly accumulated in MXZ and XYXZ.

Analysis of the variations in fatty acids using a targeted method

It is reported that fatty acids were correlated with eating quality and aroma of rice (Concepcion et al. 2018). To precisely determine the distinction of fatty acids among these three cultivars, a targeted metabolomics method (UPLC-MS/MS) was applied to measure the concentrations of fatty acids. In our study, four unsaturated fatty acids (UFA) including oleic acid, linoleic acid, palmitoleic acid, and arachidonic acid, and four saturated fatty acids (SFA) including palmitic acid, methyl palmitate, stearic acid, and behenic acid were detected. The direct quantification results with standards showed that palmitoleic acid, oleic acid, behenic acid, methyl 

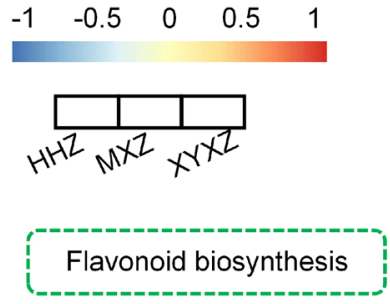

\section{Cinnamoyl-CoA}

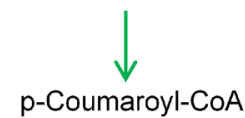

Naringenin chalcone

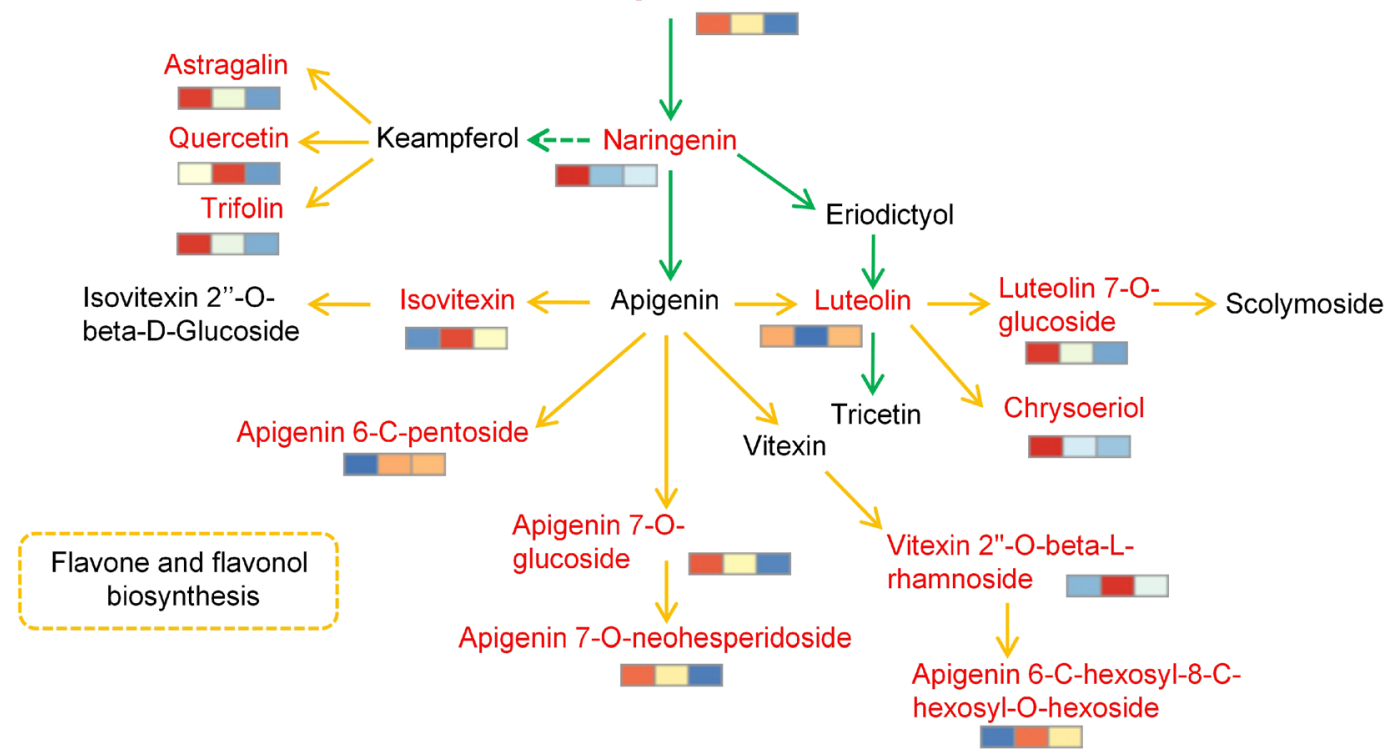

Fig. 4 Heat map of metabolites of flavonoid metabolic pathway in HHZ, MXZ, and XYXZ. This pathway is constructed based on the KEGG pathway and literary references. Each colored unit

palmitate, and stearic acid were highly accumulated in MXZ and XYXZ, especially for behenic acid and methyl palmitate which were higher in MXZ (12.99 $\mathrm{mg} \mathrm{g}^{-1}$ and $17.07 \mathrm{mg} \mathrm{g}^{-1}$ respectively) than XYXZ (5.99 $\mathrm{mg} \mathrm{g}^{-1}$ and $9.05 \mathrm{mg} \mathrm{g}^{-1}$ respectively), exceeding 2.17 and 1.89 times respectively (Fig. 6b-f). In addition, arachidonic acid was significantly increased in XYXZ compared with HHZ and MXZ (Fig. 6h). However, there were no significant differences of palmitic acid and linoleic acid among the three cultivars (Fig. 6a, g). Hence, these observations suggested that most of fatty acids were predominantly accumulated in aromatic rice $\mathrm{XYXZ}$ and MXZ. All the date is shown in Table S4.

\section{Discussion}

MXZ and XYXZ were awarded taste golden medal in the eating quality evaluation contest on account of their excellent eating quality and pursued by consumers, represents the normalized intensity of each compound according to the color scale (three biological replicates $\times$ three varieties, $n=9$ )

while HHZ is only an ordinary eating quality variety. Nevertheless, in the study, we found that it is unable to distinguish rice quality diversity of these three cultivars by traditional physicochemical characteristics. Accordingly, a widely targeted metabolomics analysis was used with brown rice of them and acquired 547 metabolites. Pairwise comparisons among cultivars with all metabolites showed that there are 86 differential metabolites between MXZ and HHZ, 109 between XYXZ and HHZ, and 71 between MXZ and XYXZ. After removing the overlapping substances, 158 differential metabolites were identified among these three cultivars (Table S5), and cluster analysis results indicated they could be divided into three subgroups, prominently accumulated in HHZ and XYXZ (class I), HHZ and MXZ (class II), and MXZ and XYXZ (class III), respectively (Fig. S1b). Compared with HHZ, there were 51 common differential compounds in MXZ and XYXZ. Of these, 30 metabolites were significantly enriched in MXZ and XYXZ, whereas 20 metabolites were 


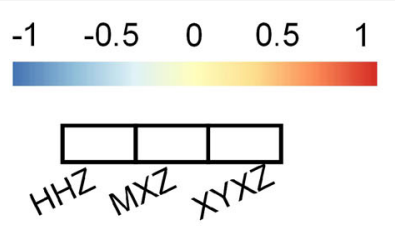

-Aspartic acid

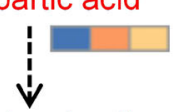

2,6-Diaminooimelic acid
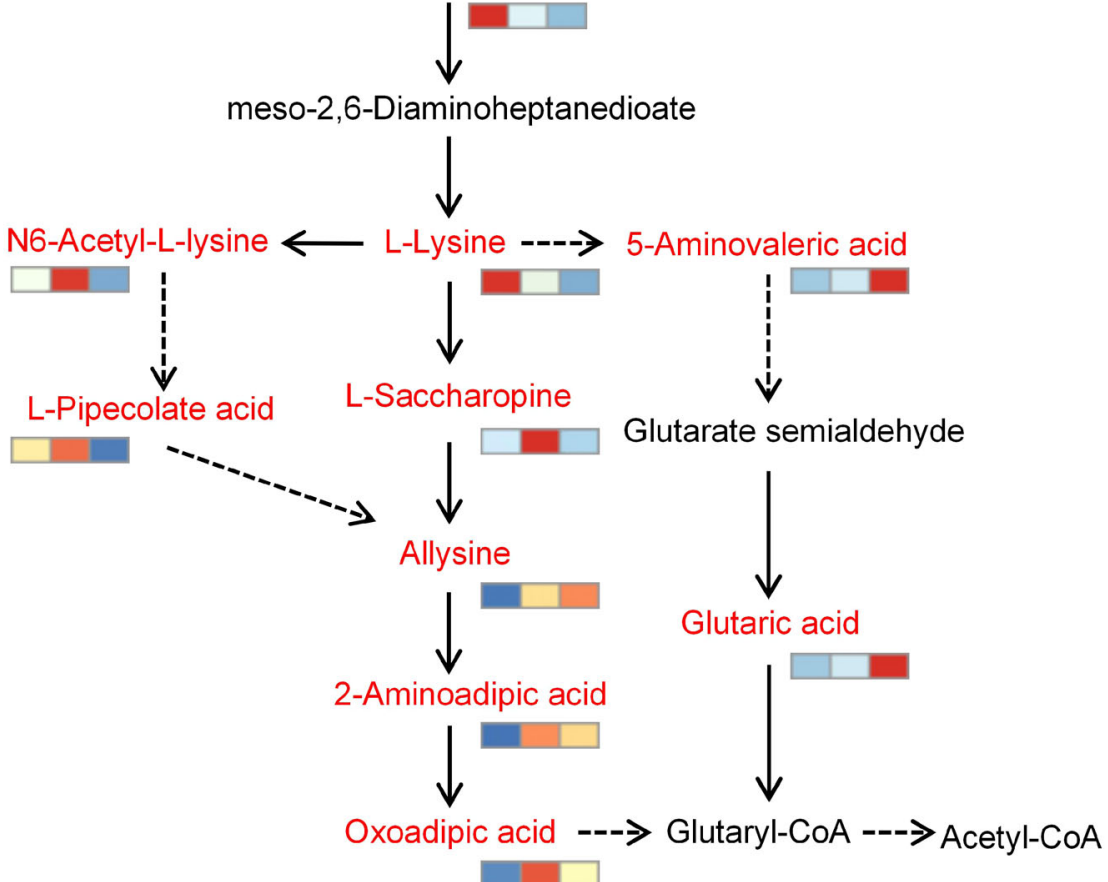

Fig. 5 Heat map of metabolites of lysine metabolic pathway in HHZ, MXZ, and XYXZ. This pathway is constructed based on the KEGG pathway. Each colored unit represents the normalized

prominently decreased compared to HHZ (Table S5), mainly including amino acids, phenolamides, flavonoids, lipids, and carbohydrates. Moreover, we found that the sample correlation between MXZ and XYXZ was much higher than that between MXZ and HHZ or XYXZ and HHZ (Fig. S2). Hence, we speculated these substances may be closely relevant to the outstanding eating quality of MXZ and XYXZ. Furthermore, we found some compounds with very high content both in MXZ and XYXZ, while could not be detected in HHZ because of the extremely low content, such as L-tryptamine, $\mathrm{N}^{\prime}, \mathrm{N}^{\prime \prime}$-diferuloylspermidine, tricin 4'-O- $(\beta-$ guaiacylglyceryl) ether 7-O-hexoside, apigenin 6-Cpentoside, and so on. In addition, some substances are with high abundance in HHZ, but cannot be found both in MXZ and XYXZ, as N-p-coumaroyl putrescine and butin. Whether these metabolites could be used as eating characteristics compounds needs further study.

Glutelin is the main storage protein which accounts for $60-80 \%$ of total protein in milled rice, and it has intensity of each compound according to the color scale (three biological replicates $\times$ three varieties, $n=9$ )

become the primary component of rice nutritional quality due to its higher lysine content and greater digestibility by human (Yang et al. 2019). We analyzed the abundance of metabolites relevant with lysine metabolism, and discovered that L-lysine and its precursor 2,6diaminoheptanedioate were enriched in HHZ. However, most of the derivative metabolites of L-lysine detected were enriched in XYXZ and MXZ, especially in MXZ. Moreover, we found that derivative allysine could not be detected in HHZ, while it has extremely high content in both MXZ and XYXZ. Allysine could be degraded to 2-aminoadipic acid under the action of 2-aminoadipate semialdehyde dehydrogenase. We detected the expression level of 2-aminoadipate semialdehyde dehydrogenase gene (LOC_Os09g26880) and found the expression level of this gene is very low in MXZ and XYXZ, but high in HHZ, consistently with the allysine accumulated levels (Fig. S3a). Previous research found that increasing lysine content in corn grains changed the expression of starch synthesis gene and affected the 


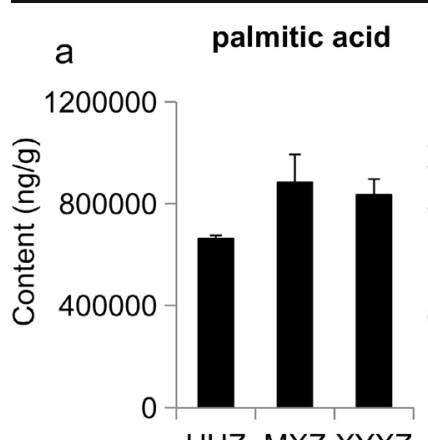

$H H Z M X Z X Y X Z$

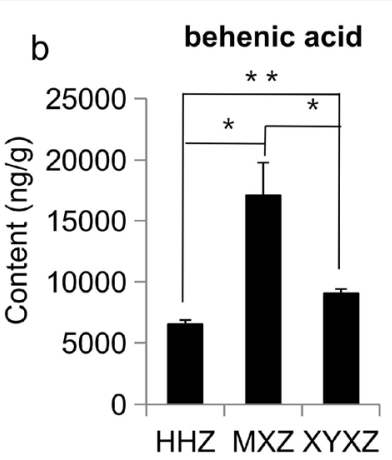

$H H Z M X Z X Y X Z$
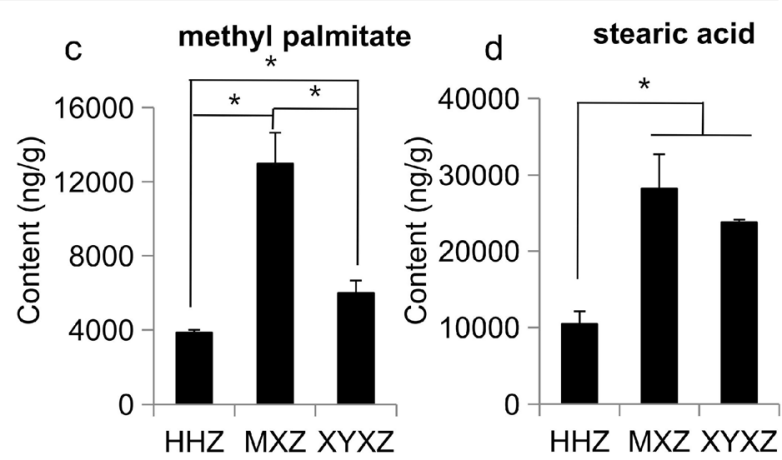
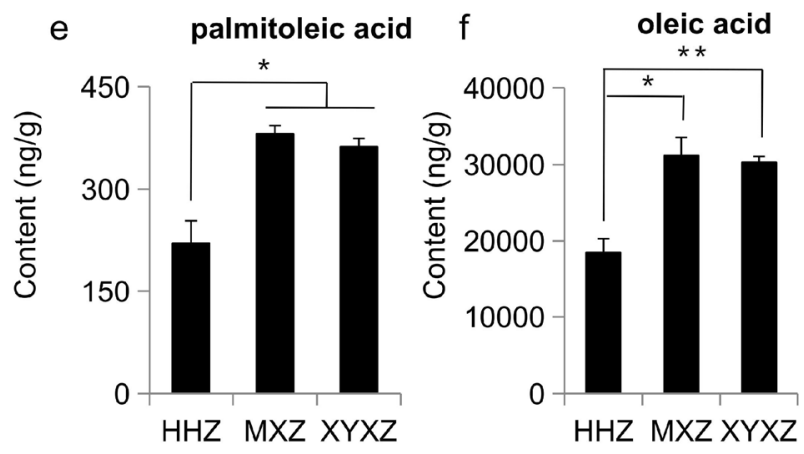

Fig. 6 Eight fatty acid contents were determined on a dry basis in HHZ, MXZ, and XYXZ. Error bars represent the standard deviation of three replicates. Date represent mean values $\pm \mathrm{SE}$ of three

physical and chemical properties of endosperm (Jia et al. 2013). In high lysine transgenic rice, the total starch content and clarity of rice grains were decreased, and the chalkiness was increased (Kawakatsu et al. 2010; Wong et al. 2015). Therefore, we speculate that the taste difference of the three cultivars may be connected with their variation in lysine metabolism.

Carbohydrates are not only important energy sources, but also the precursors of starch synthesis. As important glycogens in the central carbon metabolism, the contents of D-fructose-6-phosphate and D-glucose-6-phosphate are both remarkably elevated in XYXZ and MXZ, especially D-glucose-6-phosphate, two times higher than that in HHZ (Table S5). D-Glucose-6-phosphate generated from Dglucose-1-phosphate under the catalysis of phosphoglucomutase (PPM), and it also could be degraded into 1Dinositol-3-phosphate or 1L-inositol-1-phosphate by inositol-3-phosphate synthetase (IPS). In the study, we detected the expression of OsPPM (LOC_Os03g50480) and OsIPS (LOC_Os03g0925) and found that the expression level of $O S P P M$ in $\mathrm{HHZ}$ was significantly lower than that in MXZ and XYXZ (Fig. S3b), whereas OsIPS was prominently higher in HHZ compared with MXZ and
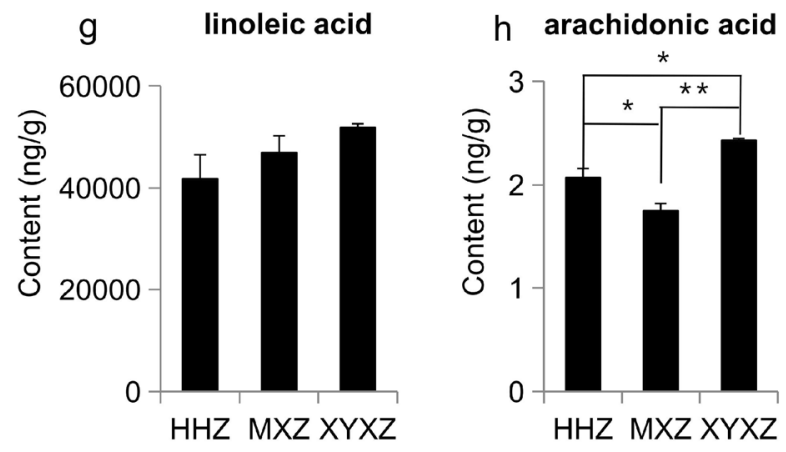

independent measurements. *and $* *$ indicate a significant difference between different varieties at $P \leq 0.05$ and $P \leq 0.01$ respectively by the Dunnett's test

XYXZ (Fig. S3c), which were consistent with the accumulation patterns of D-glucose-6-phosphate in these three cultivars. Therefore, we speculated that the high content of D-fructose-6-phosphate and D-glucose-6-phosphate in MXZ and XYXZ may be with important influence on the improvement of their eating quality.

Lipids are also an important component of rice. Although the content is low (3\% for brown rice and $0.8 \%$ for milled rice), its structure and components, such as fatty acids and phospholipids, have important impacts on the eating, appearance, and storage quality of rice (Zhou et al. 2003). It was reported that there was a significant positive correlation between the lipid content and the eating quality of rice. The rice with high lipid content had a bright appearance and better taste of cooled cooked rice (Yoon et al. 2008). Our results from widely target metabolomics showed that 19 DAMs of lipid metabolites (27\%) were detected in the three cultivars. Of these, 7 metabolites enriched both in MXZ and $\mathrm{XYXZ}$, and 10 metabolites significantly increased in MXZ or XYXZ compared with HHZ (Table S5). Moreover, we detected eight main fatty acids and found unsaturated fatty acids oleic acid and palmitoleic acid 
were increased prominently in XYXZ (improved about $64 \%$ and $65 \%$, respectively) and MXZ (improved about $69 \%$ and $73 \%$, respectively) compared with $\mathrm{HHZ}$, and arachidonic acid with a little increase in XYXZ (Fig. 6e, $\mathrm{f}, \mathrm{h}$ ). In addition, saturated fatty acids, such as methyl palmitate, stearic acid, and behenic acid, were also predominantly elevated in XYXZ and MXZ (Fig. 6b-d). Previous research indicated that unsaturated fatty acids are beneficial to human health; for example, palmitoleic acid reduces insulin resistance in diabetics and regulates insulin secretion in patients by affecting key enzymes in blood glucose metabolism (Wu et al. 2012); oleic acid and arachidonic acid can relieve excessive cholesterol in blood, enhance cell membrane permeability, and prevent myocardial block and arteriosclerosis (Yoshida et al. 2010). Furthermore, fatty acid content of rice was closely related to its palatability. It is reported that the cultivars with higher fatty acid content generally possess better eating quality (Yoon et al. 2012). In addition, unsaturated fatty acids were also explored to correlate with the formation of aroma in rice (Concepcion et al. 2018). Compared with the non-fragrant HHZ, MXZ and XYXZ are extremely aromatic cultivars. Therefore, we conjectured that these accumulated lipid metabolites were beneficial to elevate the eating quality and nutritional quality of MXZ and XYXZ.

Secondary metabolites endow rice with special nutritional value. For example, tryptamine derivatives have a variety of physiological and pharmacological activities such as anti-oxidation, antineoplastic cell, antibacterial and inhibition of melanin (Kumarasamy et al. 2003; Yamazaki et al. 2009; Csapi et al. 2010). Flavonoid intake can reduce cancer, tumor, cardiovascular disease, lipid peroxidation, and osteoporosis (Grotewold 2006). However, the effect of them to eating quality is unclear. In the study, we found the flavone C-glycosides were significantly enriched in MXZ and XYXZ compared with HHZ (Table S5). Apigenin is an important precursor of flavone $\mathrm{C}$-glycoside biosynthesis, which also possesses the biological activity of inhibiting carcinogens (Zhu et al. 2013). We explored the apigenin metabolism in these three cultivars and found that its derivatives apigenin 6-C-hexosyl-8-C-hexosyl-O-hexoside, isovitexin, apigenin 6-C-pentoside, and vitexin 2"-O-beta-L-rhamnoside were significantly enriched in MXZ and $\mathrm{XYXZ}$, especially apigenin 6-C-pentoside and apigenin 6-C-hexosyl-8-C-hexosyl-O-hexoside which could not be detected in HHZ (Fig. 4; Table S5). To our surprise, except flavone C-glycosides, $23.9 \%$ of the other flavonids' content significantly decreased both in MXZ and XYXZ, and $71.7 \%$ of them prominently reduced in MXZ or XYXZ (Table S5). Hence, we deduced that flavone $\mathrm{C}$-glycosides could be beneficial to eating quality formation, but other flavonoids detected may be with opposite effect. As the primary fragrant component, 2acetyl-1-pyrroline (2AP) makes aromatic rice with unique flavor. The 2-AP contents in XYXZ and MXZ rice are about $102 \mathrm{ng} / \mathrm{g}$ and $26 \mathrm{ng} / \mathrm{g}$ respectively (Luo et al. 2019). Except 2-AP, alcohols and aldehydes could produce different kinds of odor, which are conducive to the formation of rice aroma (Mathure et al. 2014). DAM analysis shows that there were 4 kinds of alcohol and polyol contents with significant changes in three cultivars (Table S5). Besides, we found that betaine (a kind of alkaloid) content was three times higher in MXZ and $\mathrm{XYXZ}$ than that in HHZ. Betaine is usually used as a food additive or an ideal attractant in fodder because of its unique sweetness and umami. By dietary betaine supplementation, the meat quality of chicken and duck can be significantly elevated (Wen et al. 2018; Chen et al. 2019). Hence, we speculated that these differential accumulated secondary metabolites may be relevant to rice eating quality.

With the development of social economy and the improvement of living standard, people's demand for rice quality is gradually increasing. Superior eating quality rice breeding will be an important development direction for the future seed industry. However, it is hard to accurately distinguish the rice quality level between ordinary and superior eating quality rice by traditional physicochemical characteristics. Therefore, exploring the key metabolites relevant to eating and nutritional quality by metabonomics and combining molecular detection methods would greatly accelerate the breeding process for superior eating quality rice.

Supplementary Information The online version contains supplementary material available at https://doi.org/10.1007/s11032020-01192-y.

Funding This work was supported by the Guangdong Province applied Science and Technology Research and Development Program (2015B 020231001), National High-tech Research \& Development Program of China (863 Program, 2014AA10A6), Natural Science Foundation of Guangdong Province (2019A1515011751), and Special fund for scientific innovation strategy-construction of high level Academy of Agriculture Science. 


\section{Compliance with ethical standards}

Conflict of interest The authors declare that they have no competing interests.

Open Access This article is licensed under a Creative Commons Attribution 4.0 International License, which permits use, sharing, adaptation, distribution and reproduction in any medium or format, as long as you give appropriate credit to the original author(s) and the source, provide a link to the Creative Commons licence, and indicate if changes were made. The images or other third party material in this article are included in the article's Creative Commons licence, unless indicated otherwise in a credit line to the material. If material is not included in the article's Creative Commons licence and your intended use is not permitted by statutory regulation or exceeds the permitted use, you will need to obtain permission directly from the copyright holder. To view a copy of this licence, visit http://creativecommons.org/licenses/by/4.0/.

\section{References}

Baxter G, Zhao J, Blanchard C (2010) Albumin significantly affects the pasting and textural characeristics of rice flour. Cereal Chem 87:250-255. https://doi.org/10.1094/CCHEM87-3-0250

Champagen ET, Bett-Garber KL, Thomson JL, Fitzgerald MA (2009) Unraveling the impact of nitrogen nutrition on cooked rice flavor and texture. Cereal Chem 86:274-280. https://doi. org/10.1094/CCHEM-86-3-0274

Chen FM, Zhong LJ, Wang F, Zhang GP (2005) Differences in cooking and eating properties between chalky and translucent parts in rice grains. Food Chem 90:39-46. https://doi. org/10.1016/j.foodchem.2004.03.018

Chen W, Gong L, Guo Z, Wang W, Zhang H, Liu X, Yu S, Xiong L, Luo J (2013) A novel integrated method for large-scale detection, identification, and quantification of widely targeted metabolites: application in the study of rice metabolomics. Mol Plant 6: 1769-1780. https://doi.org/10.1093/mp/sst080

Chen W, Wang W, Peng M, Gong L, Gao Y, Wan J, Wang S, Shi L, Zhou B, Li Z, Peng X, Yang C, Qu L, Liu X, Luo J (2016) Comparative and parallel genome-wide association studies for metabolic and agronomic traits in cereals. Nat Commun 7:12767. https://doi.org/10.1038/ncomms 12767

Chen R, Wen C, Cheng Y, Chen Y, Zhuang S, Zhou Y (2019) Effects of dietary supplementation with betaine on muscle growth, muscle amino acid contents and meat quality in Cherry Valley ducks. J Anim Physiol Anim Nutr 103: 1050-1059. https://doi.org/10.1111/jpn.13083

Concepcion JCT, Ouk S, Riedel A, Calingacion M, Zhao D, Ouk M, Garson MJ, Fitzgerald MA (2018) Quality evaluation, fatty acid analysis and untargeted profiling of volatiles in Cambodian rice. Food Chem 240:1014-1021. https://doi. org/10.1016/j.foodchem.2017.08.019

Csapi B, Hajdú Z, Zupkó I, Berényi Á, Forgo P, Szabó P, Hohmann J (2010) Bioactivity-guided isolation of antiproliferative compounds from Centaurea arenaria. Phytother Res 24:1664-1669. https://doi.org/10.1002 /ptr.3187

Dong T, Han R, Yu J, Zhu M, Zhang Y, Gong Y, Li Z (2019) Anthocyanins accumulation and molecular analysis of correlated genes by metabolome and transcriptome in green and purple asparaguses (Asparagus officinalis, L.). Food Chem 271:18-28. https://doi.org/10.1016/j.foodchem.2018.07.120

Fan W, Ge G, Liu Y, Wang W, Liu L, Jia Y (2018) Proteomics integrated with metabolomics: analysis of the internal causes of nutrient changes in alfalfa at different growth stages. BMC Plant Biol 18:78. https://doi.org/10.1186/s12870-018-1291-8

Fitzgerald MA, McCouch SR, Hall RD (2009) Not just a grain of rice: the quest for quality. Trends Plant Sci 14:133-139. https://doi.org/10.1016/j.tplants.2008.12.004

Gayen D, Ali N, Sarkar SN, Datta SK, Datta K (2015) Downregulation of lipoxygenase gene reduces degradation of carotenoids of golden rice during storage. Planta 242:353-363. https://doi.org/10.1007/s00425-015-2314-4

Gong L, Chen W, Gao Y, Liu X, Zhang H, Xu C, Yu S, Zhang Q, Luo J (2013) Genetic analysis of the metabolome exemplified using a rice population. Proc Natl Acad Sci U S A 110: 20320-20325. https://doi.org/10.1073/pnas.1319681110

Grotewold E (2006) The genetics and biochemistry of floral pigments. Annu Rev Plant Bio 57:761-780. https://doi. org/10.1146/annurev.arplant.57.032905.105248

Gu D, Liu Z, Liu Y, Wang S, Wang Q, Li G, Ding Y (2011) Effect of lipid content and componets on cooking quality and their responses to nitrogen in milled japonica rice. Acta Agron Sin 37: 2001-2010. https://doi.org/10.1016/S1875-2780(11)60053-9

Isshiki R, Galis I, Tanakamaru S (2014) Farinose flavonoids are associated with high freezing tolerance in fairy primrose (Primula malacoides) plants. J Integr Plant Bio 56:181188. https://doi.org/10.1111/jipb.12145

Jia M, Wu H, Clay KL, Jung R, Larkins BA, Gibbon BC (2013) Identification and characterization of lysine-rich proteins and starch biosynthesis genes in the opaque 2 mutant by transcriptional and proteomic analysis. BMC Plant Biol 13:60. https://doi.org/10.1186/1471-2229-13-60

Kang MY, Rical CW, Kim CE, Lee SC (2011) Physicochemical properities and eating qualities of milled rice from different Korean elite rice cultivars. Int J Food Prop 12:640-653. https://doi.org/10.1080/10942910903312494

Kawakatsu T, Wang S, Wakasa Y, Takaiwa F (2010) Increased lysine content in rice grains by over-accumulation of BiP in the endosperm. Biosci Biotechnol Biochem 74:2529-2531. https://doi.org/10.1271/bbb.100619

Kumarasamy Y, Middleton M, Reid RG, Nahar L, Sarker SD (2003) Biological activity of serotonin conjugates from the seeds of Centaurea nigra. Fitoterapia 74:609-612. https://doi. org/10.1016/S0367-326X(03)00144-8

Li H, Prakash S, Nicholson TM, Fitzgerald MA, Gilbert RG (2016) The importance of amylose and amylopectin fine structure for textural properties of cooked rice grains. Food Chem 196:702711. https://doi.org/10.1016/j.foodchem.2015.09.112

Liu Y, Liu J, Liu M, Liu Y, Strappe P, Sun H, Zhou Z (2019) Comparative non-targeted metabolomic analysis reveals insights into the mechanism of rice yellowing. Food Chem 308: 125621. https://doi.org/10.1016/j.foodchem.2019.125621

Livak KJ, Schmittgen TD (2001) Analysis of relative gene expression data using real-time quantitative PCR and the $2^{-\Delta \Delta C T}$ 
method. Methods 25:402-408. https://doi.org/10.1006 /meth.2001.1262

Lu Y, Lam H, Pi E, Zhan Q, Tsai S, Wang C, Ngai S (2013) Comparative metabolomics in glycine max and glycine soja under salt stress to reveal the phenotypes of their offspring. $\mathrm{J}$ Agric Food Chem 61:8711-8721. https://doi.org/10.1021 /jf402043m

Luo H, Du B, He L, He J, Tang X (2019) Exogenous application of zinc $(\mathrm{Zn})$ at the heading stage regulates 2-acetyl-1-pyrroline (2AP) biosynthesis in different fragrant rice genotypes. Sci Rep 9: 19513. https://doi.org/10.1038/s41598-019-56159-7

Mathure SV, Jawali N, Thengane RJ, Nadaf AB (2014) Comparative quantitative analysis of headspace volatiles and their association with BADH2 marker in non-basmati scented, basmati and non-scented rice (Oryza sativa L.) cultivars of India. Food Chem 142:383-391. https://doi. org/10.1016/j.foodchem.2013.07.066

Nakabayashi R, Saito K (2015) Integrated metabolomics for abiotic stress responses in plants. Curr Opin Plant Biol 24:10 16. https://doi.org/10.1016/j.pbi.2015.01.003

Saccenti E, Hoefsloot HCJ, Smilde AK, Westerhuis JA, Hendriks MM (2013) Reflections on univariate and multivariate analysis of metabolomics data. Metabolomics 10:361-374. https://doi.org/10.1007/s11306-013-0598-6

Takeuchi Y, Hori K, Suzuki K, Nonoue Y, Takemotokuno Y, Maeda H, Sato H, Hirabayashi H, Ohta H, Ishii T (2008) Major QTLs for eating quality of an elite Japanese rice cultivar, Koshihikari, on the short arm of chromosome 3 . Breed Sci 58:437-445. https://doi.org/10.1270/jsbbs.58.437

Tian Z, Qian Q, Liu Q, Yan M, Liu X, Yan C, Liu G, Gao Z (2009) Allelic diversities in rice starch biosynthesis lead to a diverse array of rice eating and cooking qualities. Proc Natl Acad Sci U S A 106:21760-21765. https://doi.org/10.1073/pnas.0912396106

Tieman D, Zhu G, Resende MFR, Lin T, Nguyen C, Bies D, Rambla LJ, Beltran KSO, Taylor M, Zhang B, Ikeda H, Liu Z, Fisher J, Zemach I, Monforte A, Zamir D, Granell A, Kirst M, Huang S, Klee H (2017) A chemical genetic roadmap to improved tomato flavor. Science 355:391-394. https://doi. org/10.1126/science.aal1556

Wakamatus KI, Sasaki O, Uezono I, Tanaka A (2008) Effect of the amount of nitrogen application on occurrence of white-back kernels during ripening of rice (Oryza Sativa) under hightemperature conditions. Jpn J Crop Sci 77:424-433. https://doi.org/10.1626/jcs.77.424

Wang X, Zhou W, Lu Z, Quyang Y, Su OC, Yao J (2015) A lipid transfer protein, OsLTPL36, is essential for seed development and seed quality in rice. Plant Sci 239:200-208. https://doi.org/10.1016/j.plantsci.2015.07.016

Wen C, Chen Y, Leng Z, Ding L, Wang T, Zhou Y (2018) Dietary betaine improves meat quality and oxidative status of broilers under heat stress. J Sci Food Agric. https://doi.org/10.1002 /jsfa.9223
Wong H, Liu Q, Sun S (2015) Biofortification of rice with lysine using endogenous histones. Plant Mol Biol 87:235-248. https://doi.org/10.1007/s11103-014-0272-z

Wu Y, Li R, Hildebrand D (2012) Biosynthesis and metabolic engineering of palmitoleate production, an important contributor to human health and sustainable industry. Prog Lipid Res 51:340 349. https://doi.org/10.1016/j.plipres.2012.05.001

Yamazaki Y, Kawano Y, Yamanaka A, Maruyama S (2009) N[(Dihydroxyphenyl)acyl] serotonins as potent inhibitors of tyrosinase from mouse and human melanoma cells. Bioorg Med Chem Lett 19:4178-4182. https://doi.org/10.1016/j. bmcl.2009.05.115

Yang Y, Guo M, Sun S, Zou Y, Yin S, Liu Y, Tang S, Gu M, Yang $Z$, Yan C (2019) Natural variation of OsGluA2 is involved in grain protein content regulation in rice. Nat Commun 10: 1949. https://doi.org/10.1038/s41467-019-09919-y

Yoon MR, Koh HJ, Kang M (2008) Variation of properties of lipid components in rice endosperm affected on palatability. J Korean Soc Appl Biol Chem 51:207-211

Yoon MR, Rico CW, Koh HJ, Kang MY (2012) A study on the lipid components of rice in relation to palatability and storage. J Korean Soc Appl Biol Chem 55:515-521. https://doi. org/10.1007/s13765-012-2050-4

Yoshida H, Tomiyama Y, Mizushina Y (2010) Lipid components, fatty acids and triacylglycerol molecular species of black and red rices. Food Chem 123:210-215. https://doi.org/10.1016 /j.foodchem.2010.04.010

Zhang Y, Chen Y, Yan G, Du B, Zhou Y, Yang J (2009) Effects of nitrogen nutrition on grain quality in uplang rice Zhonghan 3 and paddy rice Yangjing 9538 under different cultivation methods. Acta Agron Sin 35:1866-1874. https://doi. org/10.1016/S1875-2780(08)60112-1

Zhang Y, Hua J, Li Y, Chen Y, Yany J (2012) Effects of phosphorus on grain quality of upland and paddy rice under different cultivation. Rice Sci 19:135-142. https://doi. org/10.1016/S1672-6308(12)60032-8

Zhang C, Zhu J, Chen S, Fan X, Li Q, Lu Y, Wang M, Yu H, Yi C, Tang S, Gu M, Liu Q (2019) $W x^{l v}$, the ancestral allele of rice Waxy gene. Mol Plant 12:1157-1166. https://doi. org/10.3390/proceedings2019036140

Zhou Z, Blanchard C, Helliwell S, Robards K (2003) Fatty acid composition of three rice varieties following storage. J Cereal Sci 37:327-335. https://doi.org/10.1006/jcrs.2002.0502

Zhu Y, Mao Y, Chen H, Lin Y, Hu Z, Wu J, Xu X, Xu X, Qin J, Xie L (2013) Apigenin promotes apoptosis, inhibits invasion and induces cell cycle arrest of T24 human bladder cancer cells. Cancer Cell Int 13:54. https://doi.org/10.1186/14752867-13-54

Publisher's note Springer Nature remains neutral with regard to jurisdictional claims in published maps and institutional affiliations. 